\title{
Special Section Guest Editorial: Interventional and Surgical Data Science for Data-Driven Patient Outcomes
}

\author{
Amber Simpson $^{\mathrm{a}, *}$ and Michael Miga ${ }^{\mathrm{b}, *}$ \\ aQueen's University, School of Computing, Department of Biomedical and Molecular Sciences, \\ Kingston, Ontario, Canada \\ ${ }^{b}$ Vanderbilt University, Department of Biomedical Engineering, Nashville, Tennessee, \\ United States
}

This special section of Journal of Medical Imaging Volume 7, Issue 3, is focused on a new emerging area of research called interventional and surgical data science. Recognizing the long history of procedural data-science work originating from the SPIE Medical Imaging: ImageGuided Procedures, Robotic Interventions, and Modeling community, this special section called for data-driven technologies for the delivery, measurement, and monitoring of therapy. The curation of this procedural medicine data to develop best practices in surgical/interventional device development, training, planning, delivery of therapy, and post-operative care is, to a great degree, an unexplored topic with potentially profound impact on the trajectory of patient care and outcomes.

We intentionally titled the special section "Interventional and Surgical Data Science" to acknowledge the broad application of data-driven approaches to procedural medicine and to be expansive rather than reductive in our definition. However, we do draw a distinct difference as compared to other areas of healthcare data science due to the nature of data collected during intervention and surgery. While methods can certainly draw from conventional electronic medical record data, interventional and surgical data often involves a variety of temporal and spatial scales relevant to disease/dysfunction, and levels of sparsity, quality, and uncertainty that can be very difficult to anticipate. In addition, the technologies that produce these data are continually evolving with respect to measurement fidelity, delivery mechanisms, and therapeutic modality. The SPIE Medical Imaging: Image-Guided Procedures, Robotic Interventions, and Modeling community has been at the forefront of these questions since its inception over three decades ago. Only over the last decade with the emphasis on translation, has come the recognition that operating rooms and interventional suites are rich sources of data for both treatment and discovery in human systems.

\section{Special Section Paper Characteristics}

With respect to submissions for this special section drawing from this growing field, some themes did emerge. More specifically, papers reflected a need to address the variability in patient outcomes with data-driven approaches, and highlighted an intrinsic dependence between datadriving sources and procedural workflows and technology integration. Here, we detail the intersection of these themes.

\section{Addressing Variability in Patient Outcomes with Data-Driven Approaches}

The number of decisions available to a clinician that have implications for patient outcomes has massively expanded in the last two decades. Historically, patients were selected for surgery or an intervention based on anatomical considerations alone: Is the tumor resectable without causing injury to adjacent delicate structures? Will the procedure restore function? Is the anatomy endoscopically accessible? Current thinking takes into consideration a variety of other factors with complex potential consequences: Should a patient undergo a potentially morbid operation that

\footnotetext{
*Address all correspondence to Amber Simpson, E-mail: amber.simpson@queensu.ca; Michael Miga, E-mail: michael.i.miga@ vanderbilt.edu

() 2020 Society of Photo-Optical Instrumentation Engineers (SPIE)
} 
has little benefit? Will an ALK+ non-small cell lung cancer patient benefit from resection or an ALK inhibitor upfront? It is still the case that clinical decision-making is experiential and based on practice. The collection of papers in the special section intervenes in this long-held approach to determine who is the best candidate for surgery and guide surgical decision-making using data, rather than human knowledge and skill alone. De Silva et al. address variability in patient outcomes in spinal surgery by proposing an analytic framework called SpineCloud which leverages quantitative features derived from perioperative images to predict spine surgery outcome. Bakas et al. use MRI radiomics to predict survival with the eventual goal of selecting patients for potentially toxic therapies or novel clinical trials. Khan et al. analyzed surgical approach and outcomes combined with surgical guidance to reduce the risk of damaging the cochlea. Harrington et al., by contrast, determine patients that can avoid aggressive pancreatic resection by predicting the risk of malignancy in pancreatic cystic lesions using a combination of radiomics and inflammatory markers. Both Narasimhan et al. and Carton et al. augment the intraoperative environment by improving navigational assistance. These contributions share a common goal to directly influence patient outcome with analysis of preoperative and/or intraoperative data.

\section{Effects of Procedural Workflows and Technology Integration on Data-Driven Technologies}

Interestingly, all special section papers employed the most common data elements to the specific clinical application at hand with the effect of reducing the barriers to clinical adoption. Harrington et al. use imaging and cyst-fluid from endoscopic ultrasound at relevant times points in care to determine the need for resection. Carton et al. use commonly available ultrasound and preoperative MRI as input to their models. De Silva et al. rely on routinely acquired perioperative imaging for prediction model building. Narasimhan et al. track visible deforming cortical surface landmarks with standard technologies to improve neuronavigation systems. Notably, Bakas et al. remove the necessity for advanced MRI protocols (diffusion tensor imaging, dynamic susceptibility contrast, etc.) to create imaging biomarkers usable in the community, in centers without sophisticated and expensive imaging units. We note that all papers from the special section originate from large research programs with strong integration between clinical and data science teams where access to data, clinical problems, and expertise, and the physical operating rooms and interventional suites are afforded by long-standing institutional partnerships among engineering, science, and procedural medicine.

\section{Attributes of Interventional and Surgical Data Science}

Original papers were requested in topic areas specifically for the engineering of surgical and interventional systems associated with data-driven changes in procedural medicine. Authors were asked to provide a brief section called "Impact on Interventional and Surgical Data Science" describing the influence of their work on procedural decision making. While it is difficult to encapsulate all nuance to define such a burgeoning field, we reflect upon the comments of our fellow investigators to begin to shape important commonalities.

In the paper by De Silva et al., the incorporation of interventional imaging data combined with conventional electronical medical records as a means for informing surgical outcome in spine surgery is emphasized. Khan et al. use image analysis methods to measure cochlear implant integrity to create a predictive model for assisting future implants. In the work by Harrington et al., investigators use models associated with radiomic and protein data taken from CT imaging and cyst fluid analysis, respectively, to improve their patient selection for intervention. In related work, Bakas et al. propose radiomic features to predict progression-free survival in brain tumor patients to optimally assist in patient management and selection as well. In related work associated with brain tumor surgical resection, Carton et al. draw upon the medical image analysis and machine learning community to suggest population-based approaches to derive methods to better functionalize interventional imaging data, and in this case, in an attempt to provide information about tumor resection completeness during image-guided procedures. 
With a somewhat similar goal, Narasimhan et al. discuss the utilization of biophysical computational models driven by diagnostic imaging and interventional localization data to characterize and compensate for a common source of deformation that confounds image-guided brain tumor resection, namely "tissue debulking."

With this, we pose the question: "What do these applications tell us about the nature of this novel field called interventional and surgical data science?" With respect to the data itself, these applications demonstrate that data can be both highly structured as is the case of imaging data, or unstructured in the case of data taken during surgeries and interventions. To be effective, data must communicate to process in a way that is perioperatively transcendent. The applications demonstrate data at all operative stages, including historic outcomes, to influence predictive models for future procedures. Similarly, the data sources (i.e., devices and processes) and data agents (i.e., analysis and models) that make up the core of methodological realization for improving outcome are fluid, complex, of varying levels of fidelity, of differing data density, and reflect the uniqueness of the interventional/surgical theatre and respective procedural workflow. Applications demonstrate impact in patient management, stratification, and planning but also as tools to discriminate, assist, and predict outcomes of interventional and surgical events. Despite a limited sampling of applications, these precepts or, perhaps more appropriately, observations are important characteristics to this growing and exciting field. There is little doubt that they are incomplete, and yet, they do provide a developing landscape for the future.

We thank the following associate editors for their dedication and contribution to this special section:

Purang Abolmaesumi, University of British Columbia (Canada)

Matthieu Chabanas, University Grenoble (France)

Elvis C. S. Chen, Robarts Research Institute (Canada)

Sandrine de Ribaupierre, Western University (Canada)

Aaron Fenster, Robarts Research Institute (Canada)

Gabor Fichtinger, Queen's University (Canada)

Ryan Halter, Dartmouth College (United States)

David Hawkes, University College London (United Kingdom)

David R. Haynor, University of Washington (United States)

William E. Higgins, The Pennsylvania State University (United States)

David R. Holmes, Mayo Clinic (United States)

Pierre Jannin, University de Rennes 1 (France)

David M. Kwartowitz, Grand Canyon University (United States)

Shuo Li, Western University (Canada)

Kensaku Mori, Nagoya University (Japan)

Parvin Mousavi, Queen's University (Canada)

Jack H. Noble, Vanderbilt University (United States)

Maryam E. Rettmann, Mayo Clinic (United States)

Frank Sauer, Siemens Healthineers (United States)

Eric J. Seibel, University of Washington (United States)

Guy Shechter, Philips Healthcare (United States)

Jeffrey H. Siewerdsen, Johns Hopkins University (United States)

Stefanie Speidel, Karlsruher Institut für Technologie (Germany)

Tamas Ungi, Queen's University (Canada)

Satish E. Viswanath, Case Western Reserve University (United States)

Andrew D. Wiles, Northern Digital Inc. (Canada)

Ivo Wolf, Hochschule Mannheim (Germany)

Ziv R. Yaniv, National Library of Medicine (United States)

Amber Simpson is an associate professor in the School of Computing and Department of Biomedical and Molecular Sciences that specializes in biomedical data science and computer-aided surgery. Her research group is focused on developing novel computing strategies for cancer treatment. She joined the Queen's University faculty in 2019, after four years as faculty at Memorial Sloan Kettering Cancer Center in New York and three years as a Research Assistant Professor in Biomedical Engineering at Vanderbilt University in Nashville. She is 
an American Association of Cancer Research award winner and the holder of multiple National Institutes of Health grants. She received her PhD in Computer Science from Queen's University. She will continue to develop new biomarkers that improve patient care.

Michael Miga is the Harvie Branscomb Professor at Vanderbilt, and professor of Biomedical Engineering, Radiology, Neurosurgery, and Otolaryngology. He is the Director of the Biomedical Modeling Laboratory, cofounder of the Vanderbilt Institute for Surgery and Engineering, and an AIMBE Fellow. His research interests are in image/imaging guided surgery and interventions, and model-based image-data driven approaches for therapeutics. He is director of the NIH-T32 Training Program for Innovative Engineering Research in Surgery and Intervention. 